\title{
Deceptive Jamming Method with Micro-Motion Property Against ISAR
}

\author{
Ning TAI ${ }^{1}$, Chao WANG ${ }^{1}$, Liguo LIU ${ }^{2}$, Weiwei $W U^{1}$, Naichang YUAN ${ }^{1}$ \\ ${ }^{1}$ College of Electronic Science and Engineering, National University of Defense Technology, Changsha, Hunan, China \\ ${ }^{2}$ College of Electronic Engineering, Naval University of Engineering, Wuhan, Hubei, China
}

\begin{abstract}
358041578@qq.com, sywangc@163.com, poorthinker@sina.com, Shirleysp1130@ hotmail.com, yuannaichang@hotmail.com
\end{abstract}

Submitted May 3, 2016 / Accepted June 29, 2017

\begin{abstract}
Airborne target's micro-motion such as rotation or vibration causes phase modulation, termed as microDoppler effect, into radar signals. The feature of micromotion is one of the most obvious features for radar recognition in mid-course phase. In traditional works, it is assumed that the micro-motion of the scatterer is the same as the ballistic target. However, with the variation of the aspect angle of ISAR, the position of the scatterer changes. In this paper, the movement of a ballistic missile in mid-course is modeled and analyzed. A false target jamming method is proposed by combining the micro-motion modulation and the electromagnetic scattering modulation. Compared with the methods using ideal point models, our method is able to generate a vivid false target with structural information, micro-motion and variation of the scatterer's RCS. The micro-motion effect of the false target is presented through ISAR imaging and time-frequency analysis. The effectiveness and correctness of the algorithm is verified by simulation.
\end{abstract}

\section{Keywords}

Micro-motion, false target, ISAR, jammer

\section{Introduction}

Inverse synthetic aperture radar (ISAR), a remotesensing technique to provide a two-dimensional image of the interested target, has played a critical role in both military and civilian fields, such as target classification and recognition, ballistic missile defense [1], [2]. It enables the defenders with early warning, hostile target discrimination and military reconnaissance. Thus, great attention is paid to the researches on ISAR jamming and ant-jamming [3-5].

An ocean of works have been done to prevent a target from being detected by ISAR. Generally, the types of jamming methods can be classified to two categories: passive jamming and active jamming [6], [7]. Passive jamming produces jamming signals by the electromagnetic scattering from strong-reflecting objects, which do not change the prop- erties of the surrounding environments. Due to the strong reflecting ability, the scattered signal of the reflector will induce bright spot on the image of ISAR and the protected target may be smeared or submerged. A passive jamming method based on rotating reflector to produce ghost target is proposed in [8], where a netlike jamming image is achieved through the reflector array. Xu proposes a method combing micro-motion modulation and phase-switched screen (PSS), which improves the jamming ability by enlarging the jamming strip both in range direction and azimuth direction [9].

Active jamming transmits an electromagnetic signal aiming at weakening the performance of the radar. By transmitting noise-like signals with strong power, barrage jamming results in severe degradation of the ISAR image, i.e. the signal-to-noise (SNR) ratio is weakened [10], [11]. The barrage jamming needs large jamming power since the transmitted signal is non-coherent with the matched filter. So deceptive jamming method which can be coherent or partial coherent with radar matched filter attracts more and more attentions. The jamming signal should contain the information as much the same as the real target echo and it makes the target's identification to be a hard task [12]. Through false target information modulation on the radar signals, subNyquist sampling jamming signal can produce a train of false targets along the range direction [13]. With the idea of scatter-wave jamming, [14] deals with the jamming effect of sub-Nyquist jamming against ISAR where compressive sensing (CS) algorithm is applied to recover the image. It is further discussed in [15] that the situation when CS-based ISAR imaging algorithm is applied in fast-time domain to recover the high resolution range profile (HRRP).

Due to the relative movement between the radar and the target, the carrier frequency of returned radar signal will be shifted, which is known as Doppler phenomenon [16]. In addition to the bulk motion which causes a constant Doppler frequency shift, the target undergoes micro-motion dynamics, such as vibrating, rotating and rolling, which will also create time-vary frequencies [17]. The micro-motion effect is analyzed in detail by V.C. Chen in 2006 [18], [19]. The 
feature of micro-motion is academically acknowledged as one of the most important features in missile defense system. And this special characteristic is introduced in the algorithm for airborne target recognition [20] and ISAR imaging [21]. As the micro-motion will lead into additional Doppler frequency to the target echo, several researches are proposed to raise the image quality by eliminating the micro-Doppler frequency [22].

To maintain balance or interfered by external forces, airborne target, such as ballistic missile or airplane, always moves with micro-motion. Thus this phenomenon puts forward a challenge when we design a jamming method. The jamming signal should contain all the information as same as that of a real target, including micro-motion. The above false target jamming methods mainly focus on the reconstruction of the target's main body and neglect the micro-motion. The precession of a ballistic target is a typical kind of micromotion and the caused Doppler frequency is considered as a sinusoid signal in the traditional works. These researches neglect two facts: one is that the scattering center will slide along the target with the change of radar aspect angle; the other is the changing in radar cross section (RCS). The target model made up of ideal scattering centers does not concentrate on the changing of RCS. And also certain scattering center may be invisible due to the shielding effect.

In this study, we propose a jamming method which contains the micro-motion property. The modulation effects of a ballistic target with precession both in the amplitude of the scatterer are modeled and analyzed. The modulation procedure of the jamming method is introduced in detail. The false target image, where the real target information is retained, may increase the burden of ISAR while recognizing the real one.

\section{Trajectory of a Ballistic Target}

Several coordinate systems are used extensively in Orbit mechanics. The coordinates used to analyze the trajectory of a ballistic target are Earth centered inertial (ECI) coordinate, Earth centered fixed (ECF) coordinate and East, North, Up (ENU) coordinate, as shown in Fig. 1.

It is quite easy to build the dynamic model of an airborne target in ECI coordinate $o x_{I} y_{I} z_{I}$. In many tracking applications, the ENU coordinate is far more intuitive and practical than ECF or ECI coordinate. The origin of ENU coordinate is set as the location of the jammer. For simplicity, the ECI coordinate coincides with ECF coordinate at time 0 . In the following section, the location of the radar is computed in ECI coordinate and the computation of the distance between the jammer and the radar is conducted in ENU coordinate.

In ECI coordinate, the position vector is labeled as $p=\left[x_{I}, y_{I}, z_{I}\right]^{\mathrm{T}}$ and $v=\dot{p}=\left[\dot{x}_{I}, \dot{y}_{I}, \dot{z}_{I}\right]^{\mathrm{T}}$ is for the velocity vector. The status vector of a ballistic target can be defined as $X=(p, v)^{\mathrm{T}}$ and its non-linearity function is:

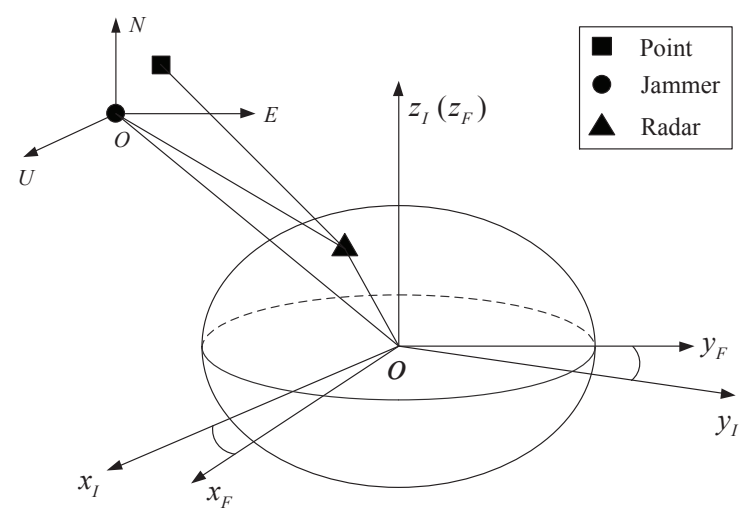

Fig. 1. Coordinates to analyze the target's movement.

$$
\dot{X}=\left[\begin{array}{c}
\dot{p} \\
\dot{v}
\end{array}\right]=\left[\begin{array}{c}
v \\
a_{\mathrm{G}}(p)
\end{array}\right]
$$

where $a_{\mathrm{G}}(p)$ is the Earth's gravity acceleration.

Considering that the flying time of the mid-course missile is quite long, we select ellipsoidal Earth model and the gravity acceleration can be [23].

$$
\begin{array}{r}
a_{\mathrm{G}}(\boldsymbol{P})=-\frac{\mu}{p^{2}}\left\{\boldsymbol{u}_{p}+\frac{3}{2} J_{2}\left(\frac{R_{\mathrm{e}}}{p}\right)^{2}\left[\left(1-5\left(\boldsymbol{u}_{p}^{\mathrm{T}} \cdot \boldsymbol{u}_{z}\right)^{2}\right) \boldsymbol{u}_{p}+\right.\right. \\
\left.\left.2\left(\boldsymbol{u}_{p}^{\mathrm{T}} \cdot \boldsymbol{u}_{z}\right) \boldsymbol{u}_{p}\right]\right\}
\end{array}
$$

where $\mu$ is the Earth gravity constant. $P$ is the vector from Earth's center to the target's location, $p=\|\boldsymbol{P}\|$ is the length of vector, $\boldsymbol{u}_{p}=\boldsymbol{P} / p$ is the unit vector and $\boldsymbol{u}_{z}$ is the unit vector along the direction of $o z_{I}$. The corresponding dynamic model of the ballistic target is [24]:

$$
\left[\begin{array}{c}
\ddot{x}_{I} \\
\ddot{y}_{I} \\
\ddot{z}_{I}
\end{array}\right]=-\frac{\mu}{r_{o}}\left[\begin{array}{c}
x_{I}+\frac{c_{\mathrm{e}}}{r_{o}^{2}}\left[1-5\left(\frac{z_{\mathrm{e}}}{r_{o}}\right)^{2}\right] x_{I} \\
y_{I}+\frac{c_{\mathrm{e}}}{r_{o}^{2}}\left[1-5\left(\frac{z_{\mathrm{e}}}{r_{o}}\right)^{2}\right] y_{I} \\
z_{I}+\frac{c_{\mathrm{e}}}{r_{o}^{2}}\left[3-5\left(\frac{z_{\mathrm{e}}}{r_{o}}\right)^{2}\right] z_{I}
\end{array}\right]
$$

where $c_{\mathrm{e}}=\frac{3}{2} J_{2} R_{\mathrm{e}}^{2}$ and $J_{2}$ is the second-order coefficient of zonal harmonics. $R_{\mathrm{e}}$ is the radius of equator. $r_{o}=\sqrt{x_{I}^{2}+y_{I}^{2}+z_{I}^{2}}$ stands for the distance from the target to the Earth's center.

We set the shut-down time of the ballistic target as the reference time, i.e. time 0 . At time $t$, a scattering point of the target in ECI coordinate can be depicted with position vector $r_{I}=\left[x_{I}, y_{I}, z_{I}\right]^{\mathrm{T}}$ and velocity vector $v_{I}=\dot{r}_{I}=\left[\dot{x}_{I}, \dot{y}_{I}, \dot{z}_{I}\right]^{\mathrm{T}}$. These two vectors in ENU coordinate are: $r_{\mathrm{r}}=\left[x_{r}, y_{r}, z_{r}\right]^{\mathrm{T}}$ and $v_{r}=\dot{r}_{\mathrm{r}}=\left[\dot{x}_{r}, \dot{y}_{r}, \dot{z}_{r}\right]^{\mathrm{T}}$. The conversion from ECI coordinate to ENU coordinate is as below:

$$
r_{\mathrm{r}}=C_{I}^{r}(t) \cdot r_{I}-\rho
$$

where $\rho$ is the vector from Earth's center to the location of the jammer in ENU coordinate

$$
\rho=\left[0,-R_{\mathrm{N}} e^{2} \sin B \cos B, R_{\mathrm{N}}+H-R_{\mathrm{N}} e^{2} \sin ^{2} B\right]^{\mathrm{T}},
$$

$C_{I}^{r}(t)$ is the conversion matrix from ECI coordinate to ENU coordinate 


$$
C_{I}^{r}(t)=\left[\begin{array}{ccc}
-\sin \varphi_{\mathrm{r}} & \cos \varphi_{\mathrm{r}} & 0 \\
-\cos \varphi_{\mathrm{r}} \sin B & -\sin \varphi_{\mathrm{r}} \sin B & \cos B \\
\cos \varphi_{\mathrm{r}} \cos B & \sin \varphi_{\mathrm{r}} \cos B & \sin B
\end{array}\right]
$$

where $\omega_{\mathrm{r}}$ is the angular velocity of Earth's rotating, $\varphi_{\mathrm{r}}=L+\omega_{\mathrm{r}} t, L, B$ and $H$ are the longitude, latitude and height of the jammer at time $0, e$ is the Earth's oblateness, $R_{\mathrm{N}}=a / \sqrt{1-e^{2} \sin ^{2} B}$ and $a$ is the semi-major axis of the reference ellipsoid.

Equation (4) turns into (7) by taking the time derivative

$$
\ddot{r}_{\mathrm{r}}=\ddot{C}_{I}^{r}(t) \cdot r_{I}+2 \dot{C}_{I}^{r}(t) \cdot \dot{r}_{I}+C_{I}^{r}(t) \cdot \ddot{r}_{I} .
$$

Equation (7) indicates that to build the dynamic equation in ENU coordinate, $\dot{r}_{I}$ and $\ddot{r}_{I}$ are needed. $\dot{r}_{I}$ and $\ddot{r}_{I}$ can be represented by the linear function which contains $\dot{r}_{\mathrm{r}}$ and $\ddot{r}_{\mathrm{r}}$ according to (4). The Runge-Kutta method can be used to solve the differential equation in (7) in ENU coordinate. Once we know the position of the jammer and the radar in real-time, the distance between them can be used to derive the modulation function which creates the false target jamming.

\section{Micro-Motion of the False Target}

\subsection{Basic of Mirco-Motion}

In traditional works, an airborne target with precession is always modeled as the isotropic scattering points, which differs from a real target. Thus when building up the model of a false target, we consider the micro-motion of the scatterer and its amplitude variation. The target's movements can be deposited into translational motion and micro-motion. Assume that the velocity of the false target is $\vec{v}$, the vector from the radar to the target's centroid $o$ is expressed as below:

$$
\vec{R}(t)=\vec{R}_{0}+\vec{v} t
$$

where $\vec{R}_{0}$ is the vector from the radar to the target at time 0 . The point $p$ on the target turns to $p_{1}$ after time $t$.

We need two coordinates named as local coordinate and velocity coordinate to analyze the motion of $P$. The details of these two coordinates will be introduced in Sec. 3.2. The location of $p$ in local coordinate is deemed as $\left(\varepsilon_{0}, \eta_{0}, \mu_{0}\right)^{\mathrm{T}}$ and the corresponding location in velocity coordinate at time $t$ is:

$$
\vec{r}_{t}=M_{t} \cdot M_{\mathrm{init}} \cdot\left(\varepsilon_{0}, \eta_{0}, \mu_{0}\right)^{\mathrm{T}}
$$

where $M_{\text {init }}$ stands for the initial Euler matrix and $M_{t}$ is the rotating matrix. So the location of $p$ in velocity coordinate is:

$$
\vec{R}(t)=\vec{R}_{0}(t)+\vec{v} t+\vec{r}_{t}
$$

Equation (9) and (10) indicate that $M_{\text {init }}$ and $M_{t}$ determine the target's moving properties. Next we will present the exact procedure to compute the micro-motion of the scatterer.

\subsection{Target Model}

The false ballistic target with precession is shown in Fig. 2. The velocity coordinate with unit vector $o X Y Z$ is set up. The origin $O$ is placed at the centroid of the false target and the direction of $O Y$ is the same as that of the false target's velocity. Another coordinate named local coordinate with unit vector $\left[e_{\epsilon}, e_{\eta}, e_{\mu}\right]^{\mathrm{T}}$ which moves with the target is also established. The $O \mu$ axis coincides with the symmetric axis of the target and points to the target's head. $O \epsilon$ and $O \eta$ reside in the plane which is normal to $O \mu$.

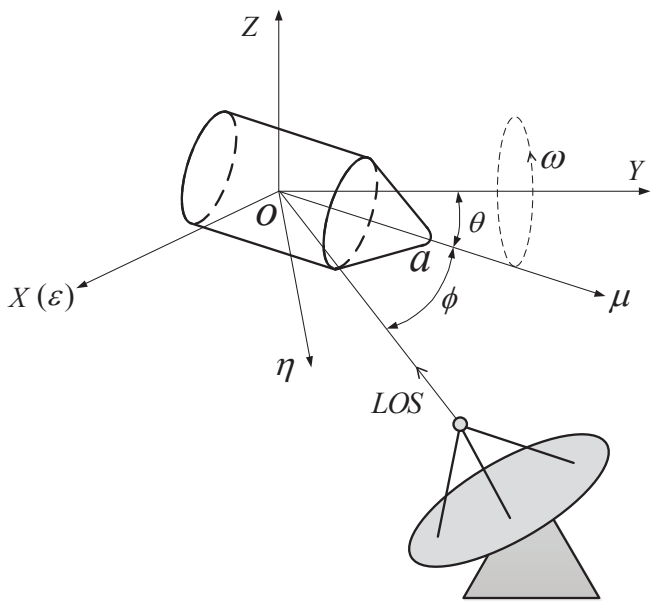

Fig. 2. False target with precession.

The target has coning motion along $O Y$ axis with angular velocity $\omega$ and spinning motion along its symmetric axis $O a$. The included angle of $O a$ and $O Y$ is $\theta$, which is the precession angle. Due to the symmetric structure, the spinning motion does not affect the position variation of scatterer. Thus only coning motion is considered in the following sections.

Firstly we analyze the distance from the radar to the jammer. Assume that at time 0, the distance from the radar to the origin of velocity coordinate is $R_{0}$, azimuth angle is $\varphi_{0}$ and pitch angle is $\theta_{0}$. The position vector of the radar is

$$
P_{b 0}=\left[R_{0} \cos \varphi_{0} \cos \theta_{0}, R_{0} \sin \varphi_{0} \cos \theta_{0}, R_{0} \sin \theta_{0}\right]^{\mathrm{T}}
$$

Then radar position vector in ENU coordinate is

$$
P_{\mathrm{R} 0}=C_{b}^{r} \cdot P_{b 0}=\left[x_{\mathrm{R} 0}, y_{\mathrm{R} 0}, z_{\mathrm{R} 0}\right]^{\mathrm{T}}
$$

where $C_{b}^{r}$ is the conversion matrix from velocity coordinate to ENU coordinate

$$
C_{b}^{r}=\left[\begin{array}{ccc}
\cos \alpha & \sin \alpha \cos \beta & -\sin \alpha \sin \beta \\
-\sin \alpha & \cos \alpha \cos \beta & -\cos \alpha \sin \beta \\
0 & \sin \beta & \cos \beta
\end{array}\right] .
$$

If the velocity of the jammer is $V_{\mathrm{J}}=\left[v_{x}, v_{y}, v_{z}\right]^{\mathrm{T}}$, then $\alpha$ and $\beta$ in (13) can be computed by: 


$$
\left\{\begin{array}{lll}
\alpha=\sin ^{-1}\left(\frac{v_{x}}{\sqrt{v_{x}^{2}+v_{y}^{2}}}\right), & -\frac{\pi}{2} \leq \alpha \leq \frac{\pi}{2}, \quad \text { if } v_{y} \geq 0 \\
\alpha=\pi-\sin ^{-1}\left(\frac{v_{x}}{\sqrt{v_{x}^{2}+v_{y}^{2}}}\right), & \frac{\pi}{2} \leq \alpha \leq \frac{3 \pi}{2}, \quad \text { if } v_{y}<0 \\
\beta=\sin ^{-1}\left(\frac{v_{z}}{\sqrt{v_{x}^{2}+v_{y}^{2}+v_{z}^{2}}}\right), & -\frac{\pi}{2} \leq \beta \leq \frac{\pi}{2}
\end{array}\right.
$$

Further we derive the distance from the radar to the jammer:

$$
R_{\mathrm{J}}(t)=\sqrt{\left(x_{\mathrm{J}}-x_{\mathrm{R} 0}\right)^{2}+\left(y_{\mathrm{J}}-y_{\mathrm{R} 0}\right)^{2}+\left(z_{\mathrm{J}}-z_{\mathrm{R} 0}\right)^{2}}
$$

where $\left[x_{\mathrm{J}}, y_{\mathrm{J}}, z_{\mathrm{J}}\right]^{\mathrm{T}}$ is the location of the jammer in ENU coordinate. The distance between the jammer and the radar is used to compute the modulation coefficient of the false target.

Next we present the micro-motion of the false ballistic target. For simplicity, the symmetric axis $\mathrm{Oa}$ resides in $Y O Z$ plane at time 0 . The Euler angle of local coordinate is $\left(\alpha_{c}, \beta_{c}, \gamma_{c}\right)=(0,-\theta-\pi / 2,0)$. So the initial conversion matrix $M_{\text {init }}$ from local coordinate to velocity coordinate can be derived

$$
M_{\text {init }}=\left[\begin{array}{ccc}
1 & 0 & 0 \\
0 & -\sin \theta & \cos \theta \\
0 & -\cos \theta & -\sin \theta
\end{array}\right] .
$$

$O a$ rotates along with $O Y$ axis with an angular velocity $\vec{\omega}$. According to Rodrigues transformation, the rotating matrix $M_{t}$ at time $t$ is

$$
M_{t}=\left[\begin{array}{ccc}
\cos \omega t & 0 & \sin \omega t \\
0 & 1 & 0 \\
-\sin \omega t & 0 & \cos \omega t
\end{array}\right]
$$

Then $O a$ at time $t$ can be expressed as:

$$
O a=[-\sin \theta \sin \omega t, \cos \theta,-\sin \theta \cos \omega t]^{\mathrm{T}} .
$$

The unit vector of light-of-sight (LOS) is

$$
\operatorname{LOS}_{\mathrm{r}}=\left[\cos \varphi_{\mathrm{r}} \cos \theta_{r}, \sin \varphi_{\mathrm{r}} \cos \theta_{r}, \sin \theta_{r}\right]^{\mathrm{T}}
$$

where

$$
\left\{\begin{array}{l}
\varphi_{r}=\sin ^{-1}\left(\frac{\left(y_{\mathrm{R} 0}-y_{\mathrm{J}}\right)}{\sqrt{\left(x_{\mathrm{R} 0}-x_{\mathrm{J}}\right)^{2}+\left(y_{\mathrm{R} 0}-y_{\mathrm{J}}\right)^{2}}}\right), \\
\theta_{r}=\sin ^{-1}\left(\frac{\left(z_{\mathrm{R} 0}-z_{\mathrm{J}}\right)}{\sqrt{\left(x_{\mathrm{R} 0}-x_{\mathrm{J}}\right)^{2}+\left(y_{\mathrm{R} 0}-y_{\mathrm{J}}\right)^{2}+\left(y_{\mathrm{R} 0}-y_{\mathrm{J}}\right)^{2}}}\right) .
\end{array}\right.
$$

The conversion matrix from ENU coordinate to velocity coordinate is $C_{\mathrm{r}}^{b}=\left(C_{b}^{r}\right)^{\mathrm{T}}$. Thus the LOS in velocity coordinate is:

$$
\operatorname{LOS}_{b}=C_{\mathrm{r}}^{b} \cdot \operatorname{LOS}_{\mathrm{r}}=\left[m_{x}, m_{y}, m_{z}\right]^{\mathrm{T}} .
$$

So the included angle of $O a$ and $L O S_{b}$ is

$$
\begin{aligned}
& \phi=\cos ^{-1}(-\sin \theta \sin \omega t \cdot m_{x}+ \\
&\left.\cos \theta \cdot m_{y}-\sin \theta \cos \omega t \cdot m_{z}\right) .
\end{aligned}
$$

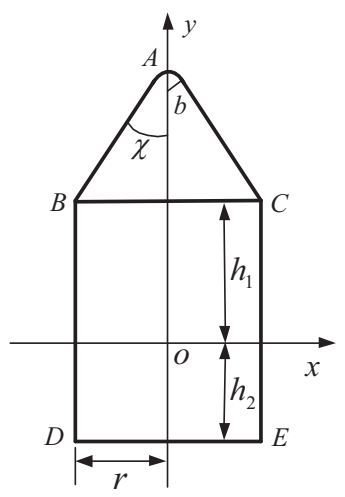

Fig. 3. Equivalent scattering points of the false target.

Assume that the plane determined by the $O a$ and $L O S$ is labeled as $\prod_{1}$. In high-frequency region, the effective scattering points of the target is the intercross points of $\prod_{1}$ and the discontinuous area of the false target. Then the scatterers can be labeled as $A, B, C, D$ and $E$, as shown in Fig. 3 .

Firstly we analyze point $A$ which is located at the head of target. According to the geometric structure, we know that:

$$
\|O A\|=h_{1}+\frac{r}{\tan \chi}+\left(1-\frac{1}{\sin \chi}\right) b .
$$

So the micro-motion of point $A$ along $L O S$ can be expressed as:

$$
\begin{aligned}
\|O A\|_{L O S} & =O A \cdot \operatorname{LOS} \\
& =\left(h_{1}+\frac{r}{\tan \chi}+\left(1-\frac{1}{\sin \chi}\right) b\right) \cos \phi .
\end{aligned}
$$

From (24) we see that the micro-motion of $A$ is determined by the aspect angle $\phi$. According to (22), $\phi$ changes periodically due to the coning motion. Next let us analyze the micro-motion of point $B$ and $C$. The normal vector of $\Pi_{1}$ is:

$$
\begin{aligned}
n_{1} & =O a \times \operatorname{LOS} \\
& =\left[\begin{array}{c}
m_{z} \cdot \cos \theta+m_{y} \cdot \sin \theta \cos \omega t \\
-m_{x} \cdot \sin \theta \cos \omega t+m_{z} \cdot \sin \theta \sin \omega t \\
-m_{y} \cdot \sin \theta \sin \omega t-m_{x} \cdot \cos \theta
\end{array}\right]
\end{aligned}
$$

where $\times$ stands for cross product.

$O B$ can be expressed as below [25]:

$$
O B=k\left(n_{1} \times O a\right)+h_{1} \cdot O a
$$

where $k$ is unknown to us so far. Considering that $n_{1}$ is perpendicular to $\mathrm{Oa}$ and $n_{1} \times O a$ is perpendicular to $O a$, we further derive

$$
\|O B\|^{2}=k^{2}\|\sin \phi\|^{2}+\left\|h_{1}\right\|^{2} .
$$

According to trigonometric function, the length of $O B$ is

$$
\|O B\|^{2}=\|r\|^{2}+\left\|h_{1}\right\|^{2} .
$$

Combining (27) and (28), $k$ can be obtained by 


$$
k= \pm\left|\frac{r}{\sin \phi}\right|
$$
$L O S$

Now we can obtain the projection of $O B$ and $O C$ on

$$
\|O B, C\|_{L O S}=(O B, C) \cdot L O S= \pm a \sin \phi+h_{1} \cdot \cos \phi
$$

where ${ }^{+}{ }^{\prime}$ refers to $O B$ and ${ }^{\prime}{ }^{\prime}$ refers to $O C$.

In the same way, the projections of points $D$ and $E$ on the LOS are:

$$
\|O D, E\|_{L O S}= \pm a \sin \phi-h_{2} \cdot \cos \phi
$$

where ${ }^{\prime}+{ }^{\prime}$ refers to $O D$ and ${ }^{\prime}-{ }^{\prime}$ refers to $O E$.

According to micro-motion of each scatter and the jammer's distance in (15) we can obtain the range distance from the scatter to the radar, which can be used to build the radar echo.

\subsection{Micro-Doppler Frequency}

For simplicity, we assume that the radar locates in the $Y O Z$ plane and the included angle of $O Y$ axis and $L O S$ is $\alpha$. The unit vector of $L O S$ in velocity coordinate can be depicted as $[0, \cos \alpha,-\sin \alpha]^{\mathrm{T}}$. So the included angle of $O a$ and $L O S$ is : $\phi=\cos ^{-1}(\cos \alpha \cos \theta+\sin \theta \sin \alpha \cos \omega t)$. Take point $D$ as an example, its Doppler frequency can be computed by

$$
\begin{aligned}
f_{\mathrm{D}} & =\frac{2 f_{\mathrm{c}}}{c} \frac{d}{d t}\left(\left\|O D_{L O S}\right\|\right) \\
& =\frac{2 f_{0} \omega \sin \alpha \sin \theta}{c} \cdot \sin \omega t \cdot\left(-h_{2}+a \frac{\cos \phi}{\sqrt{1-\cos ^{2} \phi}}\right) .
\end{aligned}
$$

According to (32), the item $(a \cdot \cos \phi) /\left(\sqrt{1-\cos ^{2} \phi}\right)$ makes the Doppler frequency be not a sinusoid function since $\phi$ varies over time. When the relative position between radar and target changes, the Doppler frequency of $D$ varies, too.

In the same way, the Doppler frequency of point $A$ is

$$
\begin{aligned}
f_{A}=\left(h_{1}+\frac{r}{\tan \chi}+\left(1-\frac{1}{\sin \chi}\right) b\right) . \\
\frac{2 f_{\mathrm{c}} \omega \sin \alpha \sin \theta}{c} \cdot \sin \omega t .
\end{aligned}
$$

From (33) we know the Doppler frequency of point $A$ is fixed under the current LOS. Its Doppler frequency is related to the size of the false target and the precession angle $\theta$. During the time of the ballistic flight, the Doppler frequency of point $A$ or $D$ will change because the LOS varies at different time.

\section{Jamming Method}

Suppose that ISAR transmits a liner frequency modulated (LFM) signal with pulse width $T_{\mathrm{p}}$, carrier frequency $f_{\mathrm{c}}$ and range chirp $k$

$$
s\left(t_{r}, t\right)=\operatorname{rect}\left(\frac{t_{r}}{T_{\mathrm{p}}}\right) \times \exp \left(\mathrm{j} 2 \pi\left(f_{\mathrm{c}} t+\frac{1}{2} k t_{r}^{2}\right)\right)
$$

where $t_{r}$ is fast time, $t_{a}$ is slow time and $t=t_{r}+t_{a}$ is full time. rect ( $\cdot)$ yields 1 when $\left|t_{r} / T_{\mathrm{p}}\right| \leq 1 / 2$ and otherwise is 0 .

Assume that the distance from one certain point to the radar is marked as $R_{i}(t)$, thus the corresponding echo in baseband is:

$$
\begin{aligned}
s_{t}\left(t_{r}, t\right)=\operatorname{rect}\left(\frac{t_{r}-2 R_{i}(t) / c}{T_{\mathrm{p}}}\right) & \times \exp \left(\mathrm { j } \pi k \left(t_{r}-\right.\right. \\
\left.\left.\frac{2 R_{i}(t)}{c}\right)^{2}\right) & \times \exp \left(-\mathrm{j} \frac{4 \pi}{\lambda} R_{i}(t)\right) .
\end{aligned}
$$

The distance from the jammer to the radar is $R_{j}(t)$, so there is a range difference $\Delta R_{i}(t)$ between $R_{i}(t)$ and $R_{j}(t)$

$$
R_{i}(t)=R_{j}(t)+\Delta R_{i}(t) .
$$

Substitute (36) into (35), we derive:

$$
\begin{aligned}
s_{t}\left(t_{r}, t\right) & =s_{j}\left(t_{r}, t\right) \otimes \delta\left(t_{r}-\frac{2 \Delta R_{i}(t)}{c}\right) \times \exp \left(-\mathrm{j} \frac{4 \pi}{\lambda} \Delta R_{i}(t)\right) \\
& =s_{j}\left(t_{r}, t\right) \otimes h\left(t_{r}, t\right)
\end{aligned}
$$

where $\delta(\cdot)$ is Dirac function and $\otimes$ means convolution. $s_{j}\left(t_{r}, t\right)$ is the radar signal intercepted by the jammer

$$
\begin{aligned}
s_{j}\left(t_{r}, t\right)=\operatorname{rect}\left(\frac{t_{r}-2 R_{j}(t) / c}{T_{\mathrm{p}}}\right) & \times \exp \left(\mathrm { j } \pi k \left(t_{r}-\right.\right. \\
\left.\left.2 R_{j}(t) / c\right)^{2}\right) & \times \exp \left(-\mathrm{j} \frac{4 \pi}{\lambda} R_{j}(t)\right) .
\end{aligned}
$$

From (37) we see that the radar echo of the scatterer $i$ can be created by the time delay and a phase addition to $s_{j}\left(t_{r}, t\right)$. $h\left(t_{r}, t\right)$ is the modulation function to produce the corresponding radar echo and it is treated as a system response function. According to the property of Fourier transform, the modulation procedure on $s_{j}\left(t_{r}, t\right)$ can be implemented by fast Fourier transform (FFT)

$$
S_{T}\left(f_{\mathrm{r}}, t\right)=S_{\mathrm{J}}\left(f_{\mathrm{r}}, t\right) \times H\left(f_{\mathrm{r}}, t\right)
$$

where $S_{T}\left(f_{\mathrm{r}}, t\right), S_{\mathrm{J}}\left(f_{\mathrm{r}}, t\right)$ and $H\left(f_{\mathrm{r}}, t\right)$ are the frequency expressions of the jamming signal, the radar signal, and the system response function, respectively

$$
H\left(f_{\mathrm{r}}, t\right)=\exp \left(-\mathrm{j} 2 \pi f_{\mathrm{r}} \frac{2 \Delta R_{i}(t)}{c}\right) \times \exp \left(-\mathrm{j} \frac{4 \pi}{\lambda} \Delta R_{i}(t)\right) .
$$

Now we obtain the frequency expression of the jamming signal after deriving $H\left(f_{\mathrm{r}}, t\right)$. Equation (40) indicates that the jamming signal is derived from the multiplication between radar signal and the system response function. And the system response function is computed according to the current position of the jammer and the movement of the false target. Knowing that the modulation is based on the intercepted 
radar signal, the induced false target after ISAR imaging is around the jammer's location.

Equation (39) turns into (41) by multiplying the scattering coefficient $\sigma$ and the inverse FFT

$$
s_{t}\left(t_{r}, t\right)=\sigma \times \operatorname{IFFT}\left(S_{\mathrm{J}}\left(f_{\mathrm{r}}, t\right) \times H\left(f_{\mathrm{r}}, t\right)\right) .
$$

Considering the false target made up of $N$ scatterers, the jamming signal is the superposition of all the echoes

$$
s_{t}\left(t_{r}, t\right)=\sum_{i=1}^{N} \sigma_{i} \times \operatorname{IFFT}\left(S_{\mathrm{J}}\left(f_{\mathrm{r}}, t\right) \times H_{i}\left(f_{\mathrm{r}}, t\right)\right) .
$$

Equation (42) indicates that the modulation process, which includes FFT, multiplication and IFFT, is conducted in parallel and $N$ times of modulation results need be summed up. Taking advantage of the linear property of FFT, the system response function of every scatterer can be summed up in advance. This work can be done when jammer is idle and the computation time is enough. The modified modulation process is

$$
s_{t}\left(t_{r}, t\right)=\operatorname{IFFT}\left(S_{\mathrm{J}}\left(f_{\mathrm{r}}, t\right) \times\left(\sum_{i=1}^{N} \sigma_{i} \times H_{i}\left(f_{\mathrm{r}}, t\right)\right)\right) .
$$

To sum up, firstly the jammer computes the real-time distance between itself and the radar. Then according to the designed micro-motion of the false target, the jammer derives the system response function. At last the radar signal and the system response function is multiplied in frequency domain to deduce the frequency expression of the jamming signal.

\section{Simulation Results}

Assume that the location of ISAR is: latitude $25^{\circ}$, longitude $31^{\circ}$ and height $100 \mathrm{~m}$. The location of the jammer at time 0 is: latitude $30^{\circ}$, longitude $10^{\circ}$ and height $100 \mathrm{~km}$. The parameters of the false target is: $b=0.08 \mathrm{~m}, h_{1}=1.6 \mathrm{~m}$, $h_{2}=0.7 \mathrm{~m}, a=0.30 \mathrm{~m}$ and $\chi=15^{\circ}$. The precession angle of the false target is set to $\theta=4^{\circ}$, angular velocity of precession is $\omega=150^{\circ} / \mathrm{s}$. The other parameters of the simulation is listed in Tab. 1.

The total flight time is about $180 \mathrm{~s}$ and it is sufficient to analyze the movements of the false target. The aspect angle changes periodically as revealed in Fig. 4. The period is $2.4 \mathrm{~s}$ since the angular velocity of the coning motion is $150^{\circ} / \mathrm{s}$. The overall trend of the aspect angle changes due to the variation of the positions of the jammer and ISAR.

\begin{tabular}{|l|l|c|}
\hline Parameter type & Value & Unit \\
\hline Carrier frequency & 10 & $\mathrm{GHz}$ \\
\hline Band width & 1 & $\mathrm{GHz}$ \\
\hline Pulse width & 0.5 & $\mu \mathrm{s}$ \\
\hline PRF & 500 & $\mathrm{~Hz}$ \\
\hline$R_{0}$ & 100 & $\mathrm{~km}$ \\
\hline$V_{\mathrm{J}}$ & 1000 & $\mathrm{~m} / \mathrm{s}$ \\
\hline
\end{tabular}

Tab. 1. Simulation parameters.

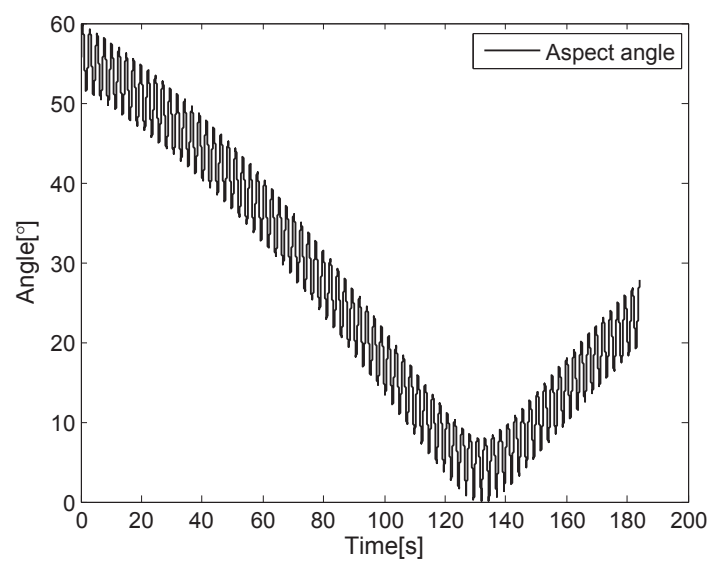

Fig. 4. Aspect angle of ISAR.

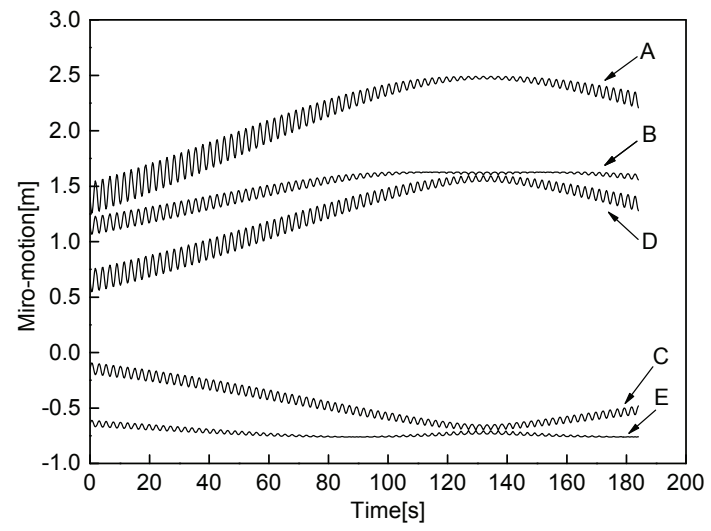

Fig. 5. Micro-motion of each scatterer.

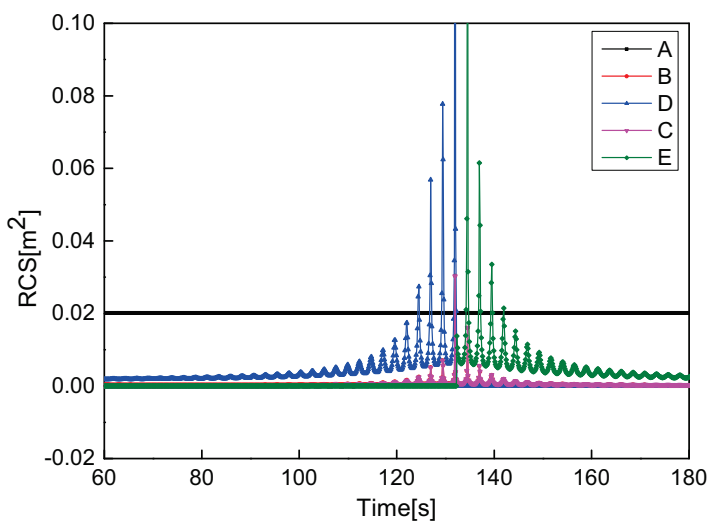

Fig. 6. RCS of each scattering point.

The micro-motion of each scattering point is demonstrated in Fig. 5. The amplitude of micro-motion changes with time and the overall trend is also related to the aspect angle. Take the movement of point $A$ as an example. When the flight time is from $0 \mathrm{~s}$ to $120 \mathrm{~s}$, the overall trend of the aspect angle decreases. So the amplitude of micro-motion of $A$ increases in this time interval, which coincides with (24). The periodically change of the amplitude is caused by the coning motion of the false target.

The RCS of each scatterer is presented in Fig. 6, where we see that the RCS of $A$ is fixed and the RCS of other scatterer is varying. Especially for point $D$ and $E$, their RCS turns into 0 in some time due to the shielding effect. Because 


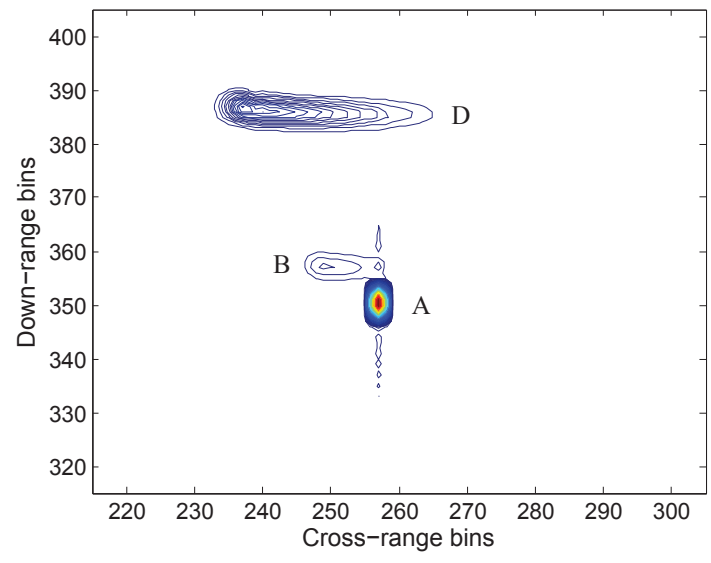

(a) Image at $60 \mathrm{~s}$.

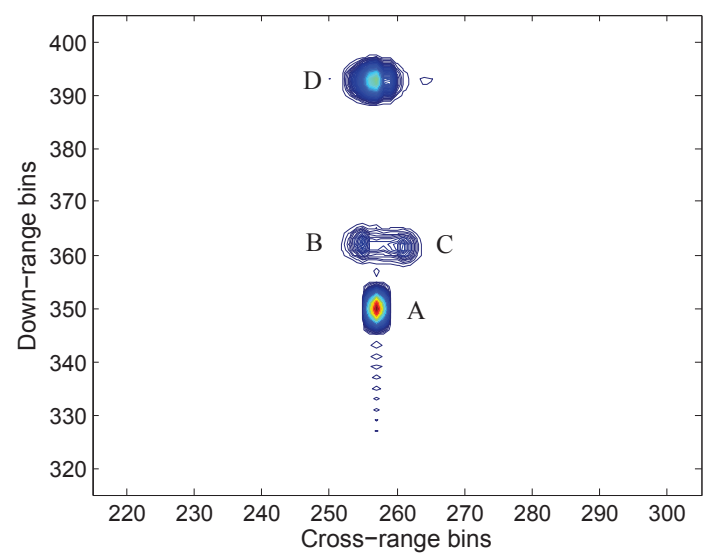

(b) Image at $120 \mathrm{~s}$.

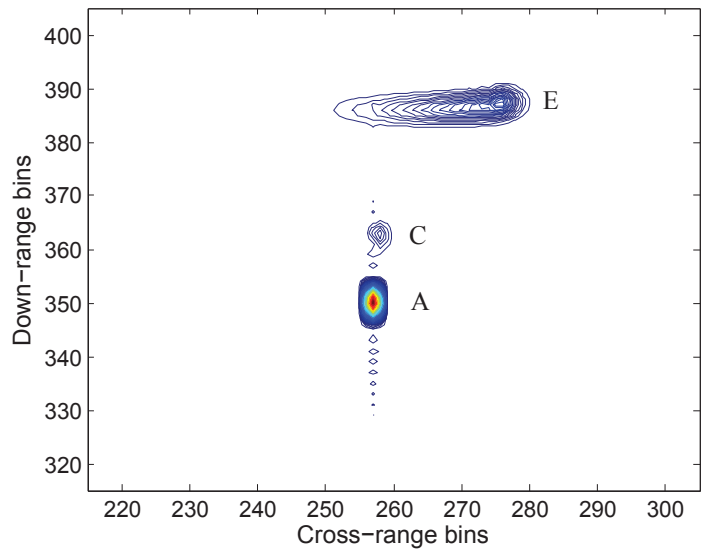

(c) Image at $180 \mathrm{~s}$

Fig. 7. ISAR image when $\theta=4^{\circ}, \omega=150^{\circ} / \mathrm{s}$.

of the symmetric structure, the RCS of $B$ is almost as same as that of $C$. The RCS of either $B$ or $C$ is small during the whole flight time except for the time interval from $110 \mathrm{~s}$ to $150 \mathrm{~s}$. According to the value of RCS, the ISAR image of the false target should be in the form of 3 focused points.

Since the Doppler frequency of the false target is varying periodically, the imaging result will be smeared along the azimuth direction. Figure 7 demonstrates the ISAR image of the jamming signal when time is $60 \mathrm{~s}, 120 \mathrm{~s}$ and $180 \mathrm{~s}$. There

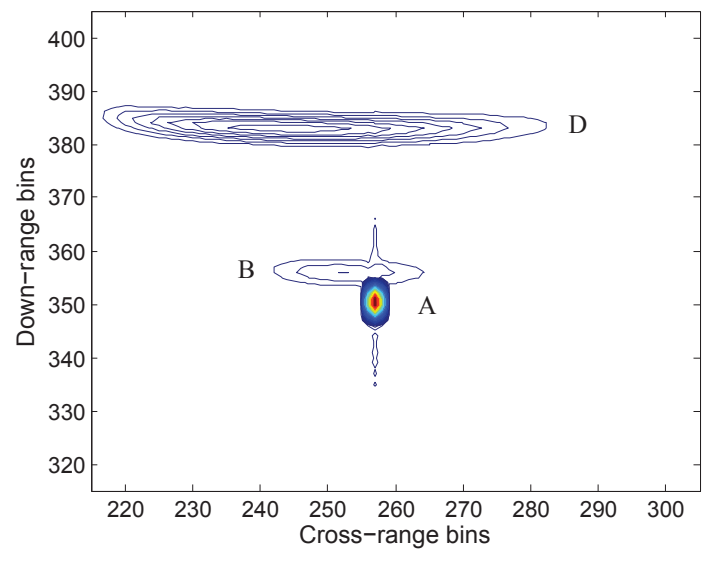

(a) Image at $60 \mathrm{~s}$

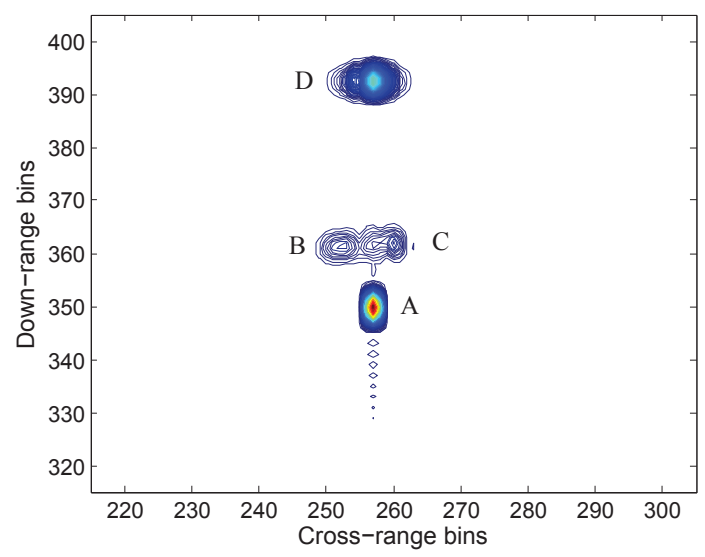

(b) Image at $120 \mathrm{~s}$.

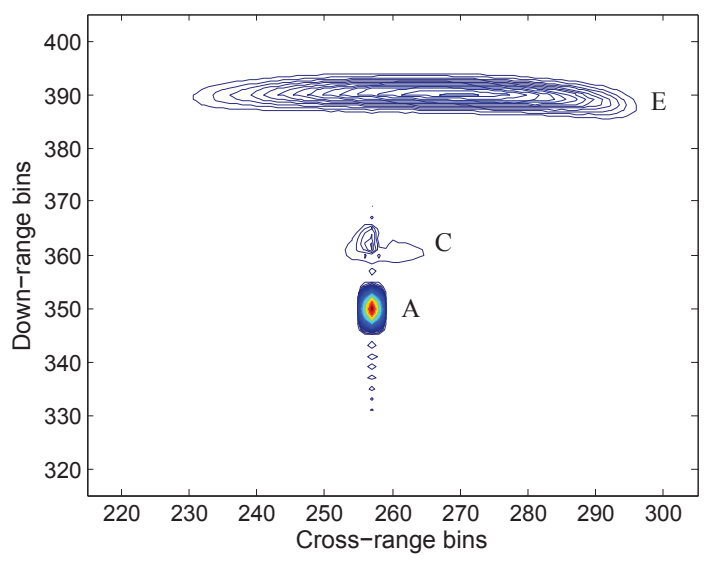

(c) Image at $180 \mathrm{~s}$.

Fig. 8. ISAR image when $\theta=10^{\circ}, \omega=120^{\circ} / \mathrm{s}$.

are 3 points to reflect the geometrical structure of the false target, which coincides with the RCS in Fig. 6. Only $A, B$ and $D$ are lighted by the radar's LOS at time $60 \mathrm{~s}$, so the result in Fig. 7a shows a target with three obvious focused points. At $120 \mathrm{~s}$, Fig. 7b shows four points because the RCS of $A, B, C$ and $D$ is relatively large at this time. Because the aspect angle of ISAR changes, the azimuth location of $D$ moves obviously when compared with the location in Fig. 7a. At this time, $B$ and $C$ are barely visible because of the small RCS. The two 


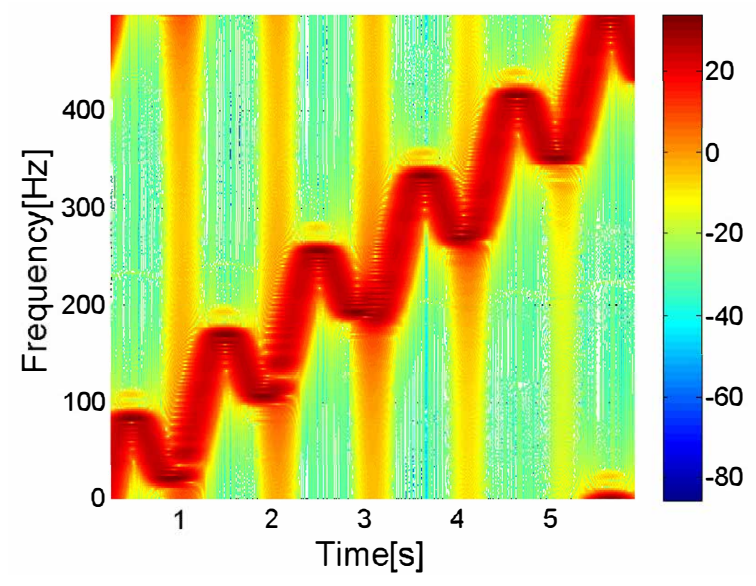

Fig. 9. STFT result of point $A$.

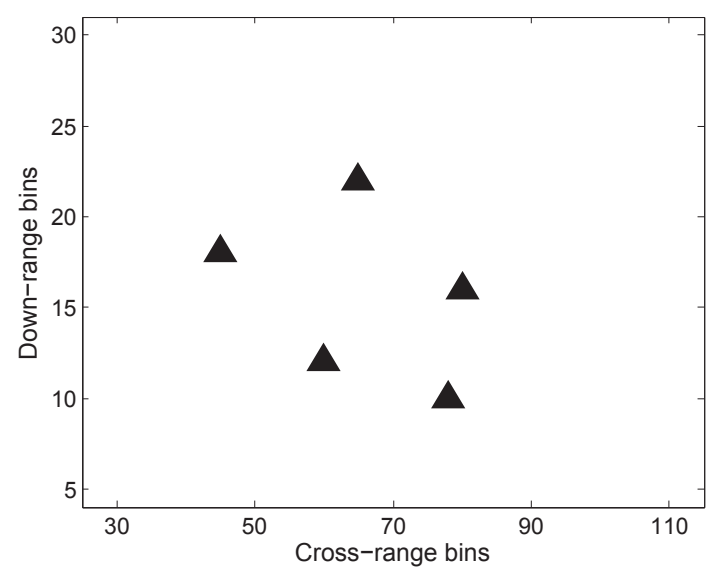

(a) Target model for DIS method.

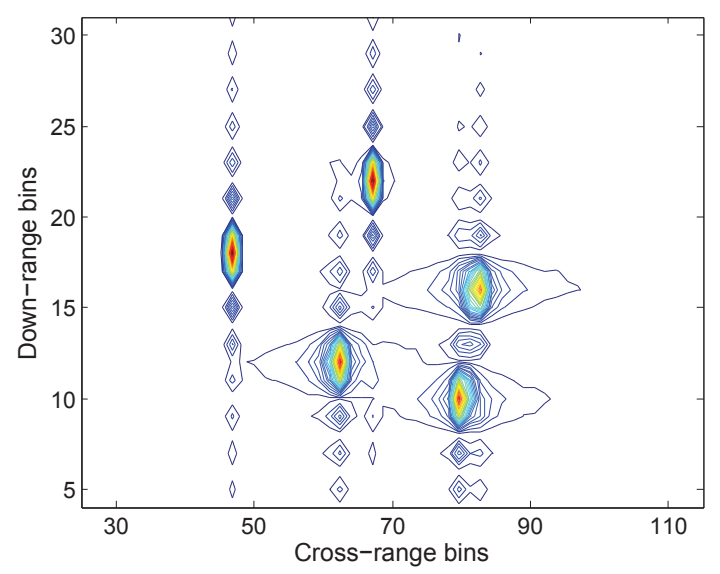

(b) ISAR image result.

Fig. 10. ISAR image of DIS method.

points are next to each other since the azimuth resolution of the ISAR image is not high enough. In Fig. 7c, $E$ appears and $D$ is invisible because of the shielding effect. The image of point $D$ or $E$ smeares along the azimuth direction, which is caused by its micro-Doppler frequency.

Another ISAR image of the jamming signal with different moving parameters is demonstrated in Fig. 8. According to (34), the Doppler frequency of a scattering point increases with a larger $\omega$ or $\theta$. The point $D$ or $E$ in Fig. 8 smears heavily than that in Fig. 9 due to it's bigger Doppler frequency, which coincides with the previous analyses in Sec. 3.3.

The features of the false target can be revealed in the time, the frequency, or the joint time-frequency domains. To analyze the time-varying Doppler frequency caused by precession, the echo of the false target is analyzed by short time Fourier transform (STFT). The observation time is $6 \mathrm{~s}$ and the STFT result of point $A$ when $\omega=360^{\circ} / \mathrm{s}$ is shown in Fig. 9. From Fig. 9 we see that the overall trend of the frequency increases with time, which is caused by the translational movement of the false target. The frequency of $A$ changes periodically due to the micro-motion and the period is $1 \mathrm{~s}$, which coincides with the conning angular velocity.

The image result of DIS method is presented here as a comparison. The range-Doppler template is demonstrated in Fig. 10a, where 5 scattering points are used to imitate a ballistic target. The amplitude of the each point is set to 1 for all range bins. The range-Doppler template is processed to generate the jamming signal according to the procedure introduced in [26]. The corresponding ISAR image is presented in Fig. 10b, where we see that the scattering points are correctly resolved.

The jamming signal created by DIS method is capable of forming an ISAR image of the designed false target, but it fails to reflect the micro-motion information of a ballistic target, which does not coincide with the real target. If the template of the false target intends to reflect the RCS of each scattering point, the extraction procedure of the modulation parameters must be processed in real time, which is not an easy job for DIS method.

\section{Conclusions}

With the fast rate of the development in ISAR technology, the electronic counter-measurement against this high resolution imaging radar becomes a hot topic in recent decades. In this paper, a jamming method which creates a ballistic false target with precession is proposed. Through transformation in ECI coordinate, ENU coordinate and velocity coordinate, the dynamic movement of the false target is modeled and the micro-motion is analyzed. It is the first time that a deceptive jamming method with micro-motion property is proposed in the mid-course flight. The Doppler frequency of the scatterer is not a sinusoid curve. The introducing of the micro-motion reflects the moving property of a ballistic target and makes the false target more similar to a real one. The micro-motion modulation of the scattering points, together with the variation of RCS, demonstrates the scattering characteristic of the false target.

When this method is applied into the engineering implementation, we recommend that the jammer adopts quadrature channels to process the intermediate frequency radar signal. To reconstruct the echo of each scatterer, the method needs frequency modulation, which requires the phase of radar sig- 
nal. Because the time-delay method is lack of precision. In our former design, the system clock is $150 \mathrm{MHz}$ and it corresponds a delay precision of 1 meter, which is not proper to reflect the characteristic of a ballistic missile. After the phase modulation, the phase of the jamming signal which represents one scatterer is transformed into amplitude. Then all the scatterers' echoes are cumulative added to build the echo of the false target.

The novelties of this paper are the considerations in the micro-motion and the variation of RCS. The false target created by our method is with a constant trace and vivid micro-motion. Compared with DIS method, the proposed method is able to imitate the constant movement of the false target and reflects the micro-motion of each scatterer. The simulation result validates the correctness of the method and the ISAR image of the jamming signal presents a false target with micro-motion property.

\section{Acknowledgments}

This work was supported in part by the National Natural Science Foundation of China under Grant no. 61302017 and 61601492. Thanks to Dr. Xi Chen for analyzing the electromagnetic scattering property of a ballistic missile. Thanks to Dr. Hong Zhu for his brilliant suggestions.

\section{References}

[1] WANG, Y. X., HAO, L., CHEN, V. C. ISAR motion compensation via adaptive joint time-frequency technique. IEEE Transaction on Aerospace and Electronic System, 1998, vol. 34, no. 2, p. 670-677. DOI: $10.1109 / 7.670350$

[2] LI, X., LIU, G. S., NI, G. L. Autofocusing of ISAR images based on entropy minimization. IEEE Transaction on Aerospace and Electronic System, 1999, vol. 35, no. 4, p. 1240-1252. DOI: $10.1109 / 7.805442$

[3] BACHMANN, D. J., EVANS, R. J., MORAN, B. Game theoretic analysis of adaptive radar jamming. IEEE Transaction on Aerospace and Electronic System, 2011, vol. 47, no. 2, p. 1081-1100. DOI: 10.1109/TAES.2011.5751244

[4] ZHANG, J., LIU, N., ZHANG., L. R., et al. Active jamming suppression based on transmitting array designation for colocated multipleinput multiple-output radar. IET Radar Sonar Navig., 2016, vol. 10, no. 3, p. 500-505. DOI: 10.1049/iet-rsn.2015.0215

[5] ALMSLMANY, A., WANG, C. Y., CAO, Q. S. Advanced deceptive jamming model based on DRFM sub-Nyquist sampling. In Proceedings of the 13th International Bhurban Conference on Applied Sciences and Technology (IBCAST), Islamabad (Pakistan), 2016, p. 727-730. DOI: 10.1109/IBCAST.2016.7429963

[6] ZHANG, R. W., LI, Y. P., JIAO, Y. F. Cognitive radar waveform diversity for anti-passive false target jamming in an active radar seeker. In Proceedings of the 15th International Conference on Instrumentation and Measurement, Computer, Communication and Control (IMCCC). Qinhuangdao (China), 2015, p. 1742-1745. DOI: 10.1109/IMCCC.2015.370

[7] LIU, Y. C., WANG, W., PAN, X. Y., et al. A frequency-domain three-stage algorithm for active deception jamming against synthetic aperture radar. IET Radar Sonar Navigation, 2014, vol. 8, no. 6 , p. 639-646. DOI: 10.1049/iet-rsn.2013.0222

[8] BAI, X. R, SUN, G. C., ZHOU, F., et al. A novel ISAR jamming method based on rotating angular reflectors. Chinese Journal of Radio Science, 2008, vol .23, no. 5, p. 867-872. DOI: $10.13443 /$ j.cjors.2008.05.019

[9] XU, L. T., FWNG, D. J., WANG, X. S. Improved synthetic aperture radar micro-Dopler jamming method based on phase-switched screen. IET Radar Sonar Navigation, 2015, vol. 10, no. 13, p. 525-534. DOI: 10.1109/JSEN.2015.2453163

[10] TAI, N., PAN, Y. J., YUAN, N. C. Quasi-coherent noise jamming to LFM radar based on pseudo-random sequence phasemodulation. Radioengineering, 2015, vol. 24, no. 4, p. 1013-1024. DOI: $10.13164 / \mathrm{re} .2015 .1013$

[11] GONG, S., WEI, X., LI, X., et al. Mathematic principle of active jamming against wideband LFM radar. Journal of Systems Engineering and Electronics, 2015, vol. 26, no. 1, p. 50-60. DOI: 10.1109/JSEE.2015.00008

[12] ZHAO, B., ZHOU, F., SHI, X. R., et al. Multiple targets deception jamming against ISAR using electromagnetic properties. IEEE Sensors Journal, 2015, vol. 15, no. 4, p. 2031-2038. DOI: $10.1109 /$ JSEN.2014.2368985

[13] XU, S. K., LIU, J. H., FU, T. W., et al. Deception jamming method for ISAR based on sub-Nyquist sampling technology. In Proceedings of the IEEE 10th International Conference on Signal Processing. Beijing (China), 2010, p. 2023-2026. DOI: 10.1109/ICOSP.2010.5655854

[14] WANG, W., PAN, X. Y., LIU, Y. C., et al. Sub-Nyquist sampling jamming against ISAR with compressive sensing. IEEE Sensors Journal, 2014, vol. 14, no. 9, p. 3131-3136. DOI: 10.1109/JSEN.2014.2323978

[15] PAN, X. Y., WANG, W., WANG, G. Y. Sub-Nyquist sampling jamming against ISAR with CS-based HRRP reconstruction. IEEE Sensors Journal, 2016, vol. 16, no. 6, p. 1597-1602. DOI: $10.1109 /$ JSEN.2015.2503419

[16] TAHMOUSH, D. Review of micro-Doppler signitures. IET Radar Sonar Navigation, 2015, vol. 9, no. 9, p. 1140-1146. DOI: 10.1049 /iet-rsn.2015.0118

[17] ZHAO, G. H., FU, T. W., NIE, L. et al. Imaging and micro-Doppler analysis of vibrating target in multi-input multi-output synthetic aperture radar. IET Radar Sonar Navigation, 2015, vol. 9, no. 9, p. 1360-1365. DOI: 10.1049/iet-rsn.2014.0480

[18] CHEN, V. C., LI, F. Y., HO, S. S., et al. Micro-Doppler effect in radar: Phenomenon, model, and simulation study. IEEE Transaction on Aerospace and Electronic System, 2006, vol. 42, no. 1, p. 2-21. DOI: 10.1109/TAES.2006.1603402

[19] CHEN, V. C. Doppler signatures of radar backscattering from objects with micro-motions. IET Signal Processing, 2006, vol. 2, no. 3 , p. 291-300. DOI: 10.1049/iet-spr:20070137

[20] COLEGROVE, S. B., DAVEY, S. J., CHEUNG, B. Separation of target rigid body and micro-Doppler effects in ISAR imaging. IEEE Transaction on Aerospace and Electronic System, 2006, vol. 42, no. 4, p. 1496-1506. DOI: 10.1109/TAES.2006.314590

[21] WANG, Y., LIN, Y. C., DAKOVIC, M., et al. ISAR imaging of nonuniformly rotating target via range-instantaneous-Doppler-derivatives algorithm. IEEE Journal of Selected Topics in Applied Earth Observations and Remote Sensing, 2014, vol. 7, no. 1, p. 167-176. DOI: 10.1109/JSTARS.2013.2257699

[22] STANKOVIC, L., THAYAPARAN, T., DAKOVIC, M., et al. MicroDoppler removal in the radar imaging analysis. IEEE Transaction on Aerospace and Electronic System, 2013, vol. 49, no. 2, p. 1234-1250. DOI: 10.1109/TAES.2013.6494410 
[23] COSTA, P. Adaptive model architecture and extended Kalman-Bucy filters. IEEE Transactions on aerospace and electronics systems, 1994, vol. 30, no. 2, p. 525-533. DOI: 10.1109/7.272275

[24] LI, X. R., JILKOV, V. P. Survey of maneuvering target tracking. Part II: Motion models of ballistic and space targets. IEEE Transactions on Aerospace and Electronic Systems, 2010, vol. 46, no. 1, p. 96-119. DOI: $10.1109 /$ TAES.2010.5417150

[25] MA, L., LIU, J., WANG, T., et al. Micro-Doppler characteristic of sliding-type scattering center on rotationally symmertric target. Science China Information Sciences, 2011, vol. 54, no. 9, p. 1957-1967. DOI: $10.1007 / \mathrm{s} 11432-011-4254-3$

[26] PACE, P. E., FOUTS, D. J., EKESTORM, S., et al. Digital false-target image synthesiser for countering ISAR. IEE Radar Sonar Navigation, 2002, vol. 149, no. 5, p. 248-257. DOI: 10.1049/ip-rsn:20020635

\section{About the Authors...}

Ning TAI was born in 1989. He received his M.Sc. from National University of Defense Technology in 2013. His research interests include radar signal processing and radar system simulation.
Chao WANG was born in 1977. He received his M.Sc. and Ph.D. from National University of Defense Technology in 2002 and 2007, respectively. His research interests include radar signal processing and microwave circuit design.

Liguo LIU was born in 1983. He received his M.Sc. and Ph.D. from National University of Defense Technology in 2008 and 2013, respectively. His research interests include the novel invisible structure and target characteristic.

Weiwei WU was born in 1981. She received his M.Sc. and Ph.D. from National University of Defense Technology in 2008 and 2011, respectively. Her research interests include antenna design and microwave circuit design.

Naichang YUAN was born in 1965. He received his M.Sc. and $\mathrm{Ph} . \mathrm{D}$. from Electronic Science and Technology from University of Electronic Science and Technology of China in 1991 and 1994, respectively. His research interests include array signal processing, SAR imaging processing and signal processing in radar. 